\title{
Medical Advancements: A Boon with Risks?
}

\author{
Yadav Karthik D* \\ Department of oral medicine and radiology, India
}

Received: 㘹July 17, 2018; Published: 畊July 25, 2018

*Corresponding author: Karthik Yadav D, Department of oral medicine and radiology, Master of Dental surgery $10^{\text {th }}$ Milestone, Bommanahalli, Hosur Road, Bangalore-560 102, India

\section{Medical Advancements: A Boon with Risks?}

Diabetes was, is and will be a major cause of concern in the healthcare sector. The control of diabetes has been a mission since a long time all over the world. The use of the glucometer has made life easier for the common man. However the lack of knowledge regarding the disposal of the glucose strips and needles has also become a matter of concern. A multi-nation study reflected that about $46.9 \%$ to $67.6 \%$ of diabetics disposed the sharps into the

Table 1: General Guidelines for Sharps Disposal.

\begin{tabular}{l} 
Option \\
\hline Drop-off \\
container \\
collection sites \\
\\
Household \\
hazardous \\
waste \\
collection sites \\
Residential \\
special waste \\
pickup services \\
Mail-back \\
programs
\end{tabular}

Syringe
exchange
programs
Home needle
destruction
devices

Description

Usually health centers, pharmacies, community organizations, police and fire stations, and medical waste facilities

Patients carry containers with sharps to these

Usually in municipalities where patients dispose sharps in sharp collection bins

Patients place special sharp containing containers to be picked up by trained sharp waste handlers Services have either regular pick up schedules or need the patient to call

Used sharps mailed to a collection sites in special containers

Usually, run by community organizations who exchange used syringes with new syringes

After destructing the needle by clipping, melting or burning through special devices, patients throw the syringe into the household trash household bins and that less than $10 \%$ used, specific containers [1]. In similar studies, improper disposal of sharps was found to be as high as $80-90 \%$ [2,3]. A study from Pakistan showed that more than $90 \%$ patients discarded them into the household bin [4]. According to the Indian Council of Medical Research, India Diabetes study circulated in 2011, revealed that about 62.4 million people in India inhabitant. were diabetics [5]. This makes India, the home to a huge diabetic
Advantages

Used sharps get separated from

the usual household garbage

Disposal in a proper manner as

medical waste

Patients can use empty

household containers

Similar to drop-off container

collection sites

Economical in the long run More control over the program

Can be utilized for both individual use and community level use Protects privacy

Ideally suited for rural

communities, remote areas, etc.

Have been found to be cost-effective, particularly in HIV prevention

Convenience

Low cost
Disadvantages

Inconvenience for the sharp user because of travel requirements Fear of losing confidentiality

Strict compliance to local regulations and biomedical safety standards may be a problem for collection sites Similar to drop-off container collection sites

Need of trained staff Start-up cost high

\section{Logistics and travel requirements} Cost of mailing may be high for some individuals

May have to face significant regulatory restrictions

Local community opposition

Puts waste workers and waste recyclers at risk of needle stick injuries
Thereby, the seriousness of the situation not only limits to diagnosing and treating the diabetic population, but it also further includes the knowledge about the proper diabetic self-care with the diet and self-monitoring of blood glucose levels forming the important part of the same. With this being said, the disposal of the waste generated from such self-monitoring techniques becomes a part of it. Thus, if neglected, it can cause substantial damage to the health of the population as well as it may reflect a marked loss to the economy, further increasing its burden. Table 1 below shows the various sharp waste disposal techniques followed [6]. According to the Environmental Protection Agency, syringes (needles) and lancets are medical wastes called "sharps." Sharps can be hazardous to those handling garbage, if the sharps are thrown in the regular waste. "Sharps boxes" are recommended for home use. Various pharmacies sell sharps boxes at a reasonable price tag and also will allow you to return the boxes when they are full $[7,8]$.

Hence, always use a sharps box for sharps disposal. Your physician may help to learn about your local disposal options and procedures. Never to re-cap your syringes before you dispose of them. In case you do not have a regular sharps box, use a hard (puncture-proof) non-clear container for disposing used clipped or 
un-clipped syringes and lancets. If you choose to clip the syringes, use a device that traps the clipped points in a puncture-proof compartment. Properly dispose of your syringes and lancets when traveling or bring your used sharps home for disposal [7,8]. Do not drop your used syringes or lancets into the regular trash. Do not cut off syringe needles with scissors or break off the needles. The needle could break off as you are cutting it and could hurt you or someone else. Do not use clear plastic bottles for syringe disposal as children or drug users may see the syringes and try to open the bottle. Do not put plastic bottles filled with syringes/lancets in recycle bin [7]. Therefore. it becomes very important to educate the individual about proper waste disposal of such sharps that are used at home to prevent any cross infection or any re-use of such materials in any manner.

\section{References}

1. Bouhanick B, Hadjadj S, Weekers L (2000) What do the needles, syringes, lancets and reagent strips of diabetic patients become in the absence of a common attitude? About 1070 questionnaires in diabetic clinics. Diabetes Metab 26(4): 288-293.

2. Costello J, Parikh A (2013) The sticking point: Diabetic sharps disposal practices in the community. J Gen Intern Med 28(7): 868-869.

3. Govender D, Ross A (2012) Sharps disposal practices among diabetic patients using insulin. S Afr Med J 102(3): 163-164.

4. Ishtiaq O, Qadri AM, Mehar S, Gondal GM, Iqbal T, et al. (2012) Disposal of syringes, needles, and lancets used by diabetic patients in Pakistan. J Infect Public Health 5(2): 182-188.

5. Anjana RM, Pradeepa R, Deepa M, Datta M, Sudha V, et al. (2011) Prevalence of diabetes and prediabetes (impaired fasting glucose and/or impaired glucose tolerance) in urban and rural India: Phase I results of the Indian Council of Medical Research-INdia DIABetes (ICMR-INDIAB) study. Diabetologia 54(12): 3022-3027.

6. Majumdar A, Sahoo J, Roy G, Kamalanathan S (2015) Improper sharp disposal practices among diabetes patients in home care settings: Need for concern? Indian Journal of Endocrinology and Metabolism 19(3): 420-425.

7. Getting Rid of Used Needles, Syringes, and Lancets. UF Health. (c) (1) This work is licensed under Creative

To Submit Your Article Click Here: Submit Article

DOI: 10.32474/MADOHC.2018.03.000151

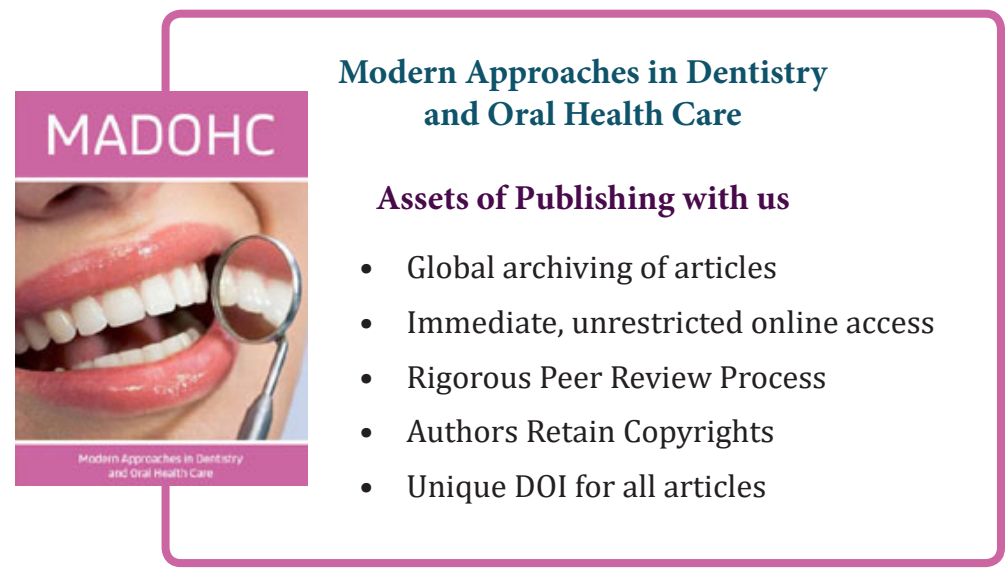

